\title{
Getting to the heart of a dealer's license
}

F or many years Dr. Georgio Martinelli, a professor at Great Eastern University, had been studying the neuroanatomy and microvascular anatomy of the canine heart. With the written permission of the animals' owners, his research used hearts from dogs that were euthanized or died naturally at the university's veterinary hospital or at a nearby not-for-profit animal shelter. Because the animals were already dead and no money changed hands, the Great Eastern IACUC never required a formal protocol from Martinelli, although the committee was fully aware of his ongoing research.

After many years of collaboration with Martinelli, the shelter began to experience financial difficulties and told Martinelli that it would have to charge him a small fee for removing the hearts from the deceased animals. Learning of this, the veterinary school also began to charge Martinelli a fee for the labor involved in removing the hearts. Concurrently, the IACUC began to reconsider if a protocol was needed, because the Animal Welfare
Act (AWA) and its regulations (AWAR) include dogs used for research, whether live or dead. Some members of the IACUC office staff said that the pertinent sections of the AWA and AWAR that mention dead animals refer to dogs or dog organs obtained from dealers, and therefore the shelter and veterinary hospital now require a dealer's license because they are charging a fee to Martinelli. They recommended that Martinelli should not be allowed to continue using those sources for hearts until the needed licenses were obtained. Furthermore, they added that a protocol was needed to assure the IACUC that Martinelli was disposing of the hearts in a manner that was consistent with $\$ 3.1$, $f$ of the AWAR.

This reasoning did not sit well with Martinelli and most IACUC members who argued that the veterinary hospital was part of the university, and an internal transfer of funds from Martinelli to the hospital was not the same as a payment to a dealer. If a not-for-profit shelter charged a small fee and therefore might be considered a dealer under the AWA, they said that was something the shelter could resolve by contacting the USDA. Either way, they felt that important research should not be hindered by a technicality. As for $\$ 3.1, \mathrm{f}$ of the AWAR, the IACUC members claimed that was an operational issue for the school, not the IACUC, and it was strange that the IACUC office never before brought that up as an issue.

The IACUC office could call the USDA and resolve the quandary, but if it is determined that a dealer's license is needed, should the IACUC prohibit Martinelli from obtaining hearts until a license is received? Does the IACUC have the authority to do that? In your opinion, is a dealer's license needed by the veterinary school?

\section{Jerald Silverman \\ University of Massachusetts Medical School, Worcester, MA, USA. \\ e-mail: Jerald.Silverman@umassmed.edu}

Published online: 24 March 2020

https://doi.org/10.1038/s41684-020-0505-5

\section{Heart is free, labor is not}

T here are four separate issues raised in this scenario concerning the acquisition of canine hearts for Dr. Martinelli's research:

1. Is a dealer's license required for the veterinary school?

2. If a dealer's license is required, should the IACUC prevent Martinelli from obtaining the hearts?

3. Does the IACUC have the authority to prevent Martinelli from obtaining hearts?

4. Does Section 3.1 of the AWAR apply to this scenario?

Two of the issues hinge on whether or not a dealer's license is required for the veterinary school. In this scenario, the veterinary school would not meet the criteria for requiring a dealer's license because the dogs are not owned by the veterinary school and the fee is for the labor in removing the hearts, not for the sale of the organs themselves. Indeed, the IACUC could use the new USDA APHIS online Animal Care Licensing and Registration Assistant to reassure themselves that a dealer's license is not required. Since a dealer's license is not required, the use of the hearts does not involve the PHS Policy on Humane Care and Use of Laboratory Animals and therefore does not involve the IACUC. The remaining two issues, therefore, are moot. The veterinary school would, of course, still need a research license with the USDA.

What if the scenario was written such that a dealer's license was required? In that case, the IACUC should prevent Martinelli from obtaining the hearts until a dealer's permit is obtained because the IACUC has the responsibility and authority to ensure that all animals used for research are obtained lawfully.

Does Section 3.1 of the AWAR - which applies to animal housing facilities-apply to the veterinary school if a dealer's license was in fact required? The veterinary school is not housing these animals; rather, they are merely collecting hearts from clientowned animals that have recently died. Section 3.1 does not apply to this situation. The disposal of the organs is an operational issue for the school and would fall under the jurisdiction of the veterinary school's department of safety.

By having a thorough understanding of the regulations the Great Eastern State Veterinary School's IACUC can keep their institution in full compliance, and prevent unnecessarily hindering research.

Leslie Birke ${ }^{凶}$ and Adam Caro

Division of Animal Care, LSU Health Sciences

Center, New Orleans, LA, USA.

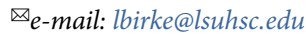

Published online: 24 March 2020

https://doi.org/10.1038/s41684-020-0507-3 